\title{
BMJ Open Interaction of sleep quality and sleep duration on impaired fasting glucose: a population-based cross-sectional survey in China
}

\author{
Peian Lou, Peipei Chen, Lei Zhang, Pan Zhang, Guiqiu Chang, Ning Zhang, \\ Ting Li, Cheng Qiao
}

To cite: Lou $P$, Chen $P$, Zhang $L$, et al. Interaction of sleep quality and sleep duration on impaired fasting glucose: a population-based cross-sectional survey in China. BMJ Open 2014:4: e004436. doi:10.1136/ bmjopen-2013-004436

- Prepublication history for this paper is available online. To view these files please visit the journal online (http://dx.doi.org/10.1136/ bmjopen-2013-004436).

Received 8 November 2013 Revised 12 February 2014 Accepted 13 February 2014

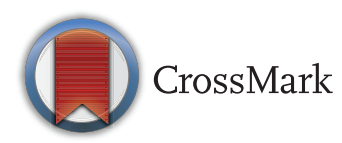

Department of Control and Prevention of Chronic Noncommunicable Diseases, Xuzhou Center for Disease Control and Prevention, School of Public Health, Xuzhou Medical University, Xuzhou, China

\section{Correspondence to} Dr Peian Lou; Ipa82835415@ 126.com

\section{ABSTRACT}

Objectives: To explore the interactions of sleep quality and sleep duration and their effects on impaired fasting glucose (IFG) in Chinese adults.

Design: Cross-sectional survey.

Setting: Community-based investigation in Xuzhou, China.

Participants: 15145 Chinese men and women aged 18-75 years old who fulfilled the inclusion criteria.

Primary and secondary outcome measures: The Pittsburgh Sleep Quality Index was used to produce sleep quality categories of good, common and poor. Fasting blood glucose levels were assessed for IFG. Sleep duration was measured by average hours of sleep per night, with categories of $<6,6-8$ and $>8 \mathrm{~h}$. The products of sleep and family history of diabetes, obesity and age were added to the logistic regression model to evaluate the addictive interaction and relative excess risk of interaction (RERI) on IFG. The attributable proportion (AP) of the interaction and the synergy index (S) were applied to evaluate the additive interaction of two factors. Bootstrap measures were used to calculate $95 \% \mathrm{Cl}$ of RERI, AP and S.

Results: The prevalence of IFG was greatest in those with poor sleep quality and short sleep duration (OR $6.37,95 \% \mathrm{Cl} 4.66$ to 8.67 ; $\mathrm{p}<0.001$ ) compared with those who had good sleep quality and 6-8 $\mathrm{h}$ sleep duration, after adjusting for confounders. After adjusting for potential confounders RERI, AP and $S$ values (and their $95 \% \mathrm{Cl}$ ) were 1.69 ( 0.31 to 3.76$), 0.42$ (0.15 to 0.61 ) and 2.85 (2.14 to 3.92 ), respectively, for the interaction between poor sleep quality and short sleep duration, and 0.78 ( 0.12 to 1.43 ), 0.61 (0.26 to 0.87 ) and $-65(-0.94$ to -0.27$)$ for the interaction between good sleep quality and long sleep duration.

Conclusions: The results suggest that there are additive interactions between poor sleep quality and short sleep duration.

\section{INTRODUCTION}

Prediabetes has a prevalence of $15.5 \%$ and affects an estimated 148.2 million Chinese

\section{Strengths and limitations of this study}

- The strengths of this study were that participants of a large sample were randomly selected from the general population of Xuzhou, and many confounding risk factors were adjusted for.

- Owing to the cross-sectional design, we could not determine a causal relationship between sleep quality, sleep duration and impaired fasting glucose (IFG).

- We were not able to control for some important and well-known risk factors of diabetes-for example, snoring.

- We did not measure poor diet, which is causally related to type 2 diabetes and may also influence sleep patterns.

adults. ${ }^{1}$ Prediabetes, including impaired fasting glucose (IFG) and impaired glucose tolerance (IGT), is the most important risk factor for type 2 diabetes. The average annual incidence of diabetes in a cohort of patients with IFG is $11 \%$ over a 6 -year period without intervention. ${ }^{2}$ Older age, a family history of diabetes, overweight, obesity, central obesity, increased heart rate, elevated systolic blood pressure, elevated serum triglyceride levels, high income, history of hypertension, history of coronary heart disease, history of drinking, eating pickled foods and low educational level are significantly associated with an increased risk of prediabetes. ${ }^{13}$ Increasingly, studies have shown that prediabetes is associated with poor sleep quantity and quality. ${ }^{3}{ }^{4} \mathrm{~A}$ cross-sectional study has revealed that, compared with those sleeping $7-8 \mathrm{~h}$ per night, individuals aged $<60$ years who slept $5 \mathrm{~h}$ or less had an increased OR for IGT (OR 1.37, 95\% CI 1.13 to 1.67 ). ${ }^{4}$ The Western New York Health Study including 1455 participants showed that sleep duration of less than $6 \mathrm{~h}$ was associated with 
increased prevalence of IFG, compared with those sleeping $6-8 \mathrm{~h} /$ night, after adjustment for confounders, over 6 years of follow-up. ${ }^{5}$ Spiegel et $a l^{6}$ have demonstrated that experimental restriction of sleep to $<4 \mathrm{~h} /$ night for six nights resulted in an IGT in young healthy adults.

Our previous results suggest that poor quality of sleep and sleep duration of $<6 \mathrm{~h} /$ night are independent risk factors for type 2 diabetes, even after adjusting for a large number of possible confounders. ${ }^{78}$ Our previous study also confirmed that relatively healthy individuals with poor sleep quality and sleeping times of $6 \mathrm{~h}$ or less had a higher risk of IFG, even after adjusting for a large number of confounding factors. ${ }^{3}$

Although these risk factors play a role in the development of type 2 diabetes, the disease is the result of the interaction of genetic and environmental factors. There is little understanding of multivariate explanations of IFG in relatively healthy individuals. To our knowledge, there are no studies on the interaction of sleep quality and sleep duration on IFG in relatively healthy individuals. The primary aim of this cross-sectional study was to examine the combined effects of sleep quality and sleep duration on IFG in relatively healthy individuals in a Chinese primary-care setting. A secondary aim was to assess the associations of sleep quality and IFG, and of sleep duration and IFG.

\section{METHODS}

The study was a continuation of our previous work. ${ }^{9}$ The investigation was conducted from March to November 2012 with a sample size of 15145 volunteers (7557 men and 7588 women) aged $18-75$ years. Briefly, the sampling was selected with probability proportional to size from all of the 11 regions in Xuzhou city. In the first stage, five subdistricts/townships in urban/rural areas were selected from each region. In the second stage, five communities/villages were selected from each subdistricts/townships. In the final stage, one person who was at least 18 years old and lived in the current residence for at least 5 years was selected from each household using a Kish selection table. A total of 16500 people were selected assuming an estimation prevalence of diabetes of $5.5 \%$ with $90 \%$ power and $\alpha=0.05$ and allowing for a drop out of $10 \%$. All volunteers underwent a health check and completed a structured questionnaire covering demographic information, medical history, medication history, sleep assessment, and smoking, alcohol drinking and exercise habits. All volunteers underwent $12 \mathrm{~h}$ overnight fasting and blood sampling for basic fasting plasma glucose (FPG). After blood sampling, each volunteer completed the Pittsburgh Sleep Quality Index (PSQI) ${ }^{10}$ We excluded volunteers who were pregnant, had received antihypertensive medication, or were suffering from any cardiovascular disease, stroke, neuropathy, psychosis, depression, chronic obstructive pulmonary disease, obstructive sleep apnea, diabetes, ache or any other disease. Those who had missing information on sleep duration or sleep quality were also excluded. Trained physicians and public health workers conducted face-to-face interviews using a standardised questionnaire to collect sociodemographic, lifestyle and health-related information.

The study protocol was approved by the Xuzhou Center for Disease Control and Prevention.

\section{Key measurements}

The FPG was determined by morning blood samples obtained by venipuncture after an overnight fast of at least $12 \mathrm{~h}$, and extracted plasma was stored at $-70^{\circ} \mathrm{C}$ for later glucose determination by the hexokinase method. IFG is delimited according to the current American Diabetes Association definition. ${ }^{11}$

The PSQI is a 19-item self-report measure of sleep quality and degree of sleep difficulties over the past month, and contains 7 component scales: sleep quality, sleep latency, sleep duration, sleep efficiency, sleep disturbances, use of sleep medication, and daytime dysfunction. The 7 component scores are then added to yield a global PSQI score with a range of 0-21; higher scores indicate worse sleep quality. A global PSQI score $>5$ has a diagnostic sensitivity of $89.6 \%$ and specificity of $86.5 \%$ in differentiating poor from good sleepers. The Chinese version of the PSQI, used with permission from the original PSQI authors, has an overall reliability coefficient of $0.82-0.83$ and acceptable test-retest reliability, with a coefficient of $0.77-0.85 .{ }^{12}$ Accordingly, in this study design, a PSQI score $\leq 5$ was also conventionally defined as 'good sleep quality', and a PSQI score $>5$ was defined as 'poor sleep quality'.

Self-reported sleep measures of chronic sleep: two variables were used to evaluate degree of 'chronic sleep restriction' by estimating average nightly sleep duration: (1) 'usual sleep' (from questionnaires) and (2) 'average nightly sleep' (from sleep diaries). Sleep quantity was categorised as $<6,6-8$ and $>8 \mathrm{~h} /$ night in accordance with our previous study. ${ }^{3} 8$

\section{Covariates}

Age, gender, current employment status, level of education, cigarette smoking, alcohol intake, physical activity, family history of diseases including diabetes, hypertension, heart disease and cancer were assessed using a standardised questionnaire. Employment status was categorised as manual, non-manual, unemployed and retired. Education was categorised into below high school, high school or above high school education. Lifestyle variables included cigarette smoking, alcohol drinking and physical activity level. Cigarette smoking was defined as having smoked at least 100 cigarettes in a lifetime. Information was obtained on the amount and type of alcohol that was consumed during the previous year, and alcohol drinking was defined as the consumption of at least $30 \mathrm{~g}$ of alcohol per week for 1 year or more. Regular leisure-time physical activity was defined as participating in moderate or vigorous activity for no less than $30 \mathrm{~min} /$ day at least 3 days a 
Table 1 Baseline characteristics of the study population ( $\mathrm{N}=15$ 145)

\begin{tabular}{|c|c|c|c|c|}
\hline \multirow[b]{2}{*}{ Reported variable } & \multirow[b]{2}{*}{ All } & \multicolumn{3}{|l|}{ IFG } \\
\hline & & $\overline{\text { No }}$ & Yes & p Value \\
\hline & 15145 & $14511(95.81 \%)$ & $634(4.19 \%)$ & \\
\hline Age (years) & $47.6 \pm 15.1$ & $47.4 \pm 14.7$ & $52.2 \pm 16.2$ & 8.012 \\
\hline$\geq 45$ years & 8362 & $7928(94.81 \%)$ & $434(5.19 \%)$ & 0.000 \\
\hline$\overline{\text { Sex }}(\%$ male $)$ & 49.9 & 50 & 49.8 & 0.938 \\
\hline Rural (\%) & 72.4 & 72.4 & 72.9 & 0.795 \\
\hline Manual & 10833 & 10359 (95.62\%) & $474(4.38 \%)$ & 0.023 \\
\hline Non-manual & 1045 & $1016(97.22 \%)$ & $29(2.78 \%)$ & \\
\hline Unemployed & 677 & $658(97.19 \%)$ & $19(2.81 \%)$ & \\
\hline Retired & 2590 & $2478(95.68 \%)$ & $112(4.32 \%)$ & \\
\hline Marred (living with partners; \%) & 88.5 & 88.6 & 86.3 & 0.073 \\
\hline Below high school & 11899 & $11400(95.81 \%)$ & 499 (4.19\%) & 0.986 \\
\hline High school & 1760 & $1686(95.80 \%)$ & $74(4.20 \%)$ & \\
\hline Above high school & 1486 & $1425(95.90 \%)$ & $61(4.10 \%)$ & \\
\hline Smoker & 3541 & 3367 (95.09\%) & $174(4.91 \%)$ & 0.014 \\
\hline Alcohol use & 2872 & 2752 (95.82\%) & $120(4.18 \%)$ & 0.981 \\
\hline Regular exercise & 2559 & $2452(95.82 \%)$ & $107(4.18 \%)$ & 0.989 \\
\hline Family history of diabetes & 483 & $362(74.95 \%)$ & $121(25.05 \%)$ & 0.000 \\
\hline BMI, mean (SD) & $23.9 \pm 4.7$ & $23.8 \pm 5.8$ & $25.2 \pm 4.1$ & $<0.001$ \\
\hline Central obesity & 4613 & $4219(91.46 \%)$ & 394 (8.54\%) & 0.000 \\
\hline Hypertension & 3060 & $2901(94.80 \%)$ & $159(5.20 \%)$ & 0.002 \\
\hline \multicolumn{5}{|l|}{ Sleep duration (h) } \\
\hline$\leq 6$ & 1984 & 1839 (92.70\%) & $145(7.30 \%)$ & 0.000 \\
\hline $6-8$ & 11292 & $10919(96.70 \%)$ & $373(3.30 \%)$ & \\
\hline$\geq 8$ & 1869 & $1753(93.80 \%)$ & $116(6.20 \%)$ & \\
\hline \multicolumn{5}{|l|}{ Sleep quality } \\
\hline Good & 11209 & $10840(96.71 \%)$ & $369(3.29 \%)$ & 0.000 \\
\hline Poor & 3936 & $3671(93.27 \%)$ & $265(6.73 \%)$ & \\
\hline
\end{tabular}

week. Each volunteer's body height (to the nearest $0.1 \mathrm{~cm}$ ) and weight (to the nearest $0.1 \mathrm{~kg}$ ) in light indoor clothing were measured. Body mass index (BMI; in $\mathrm{kg}$ / $\mathrm{m}^{2}$ ) was calculated. BMI was categorised as underweight $\left(<18.5 \mathrm{~kg} / \mathrm{m}^{2}\right)$, normal weight $\left(18.5-24 \mathrm{~kg} / \mathrm{m}^{2}\right)$ and overweight/obese $\left(>24 \mathrm{~kg} / \mathrm{m}^{2}\right) .{ }^{13}$

\section{Statistical analysis}

Statistical analysis was performed on a computer, using the statistical analysis program SPSS V. 13.0 (SPSS, Chicago, Illinois, USA). Mean differences of continuous variables between groups were tested using analysis of variance. The $\chi^{2}$ - test was used to calculate the difference in proportions between groups. Logistic regression analysis was performed to estimate the probability of having IFG and 95\% CI for each risk factor category stratified by sleep quality and sleep duration, adjusting for age, residential areas, occupation, education and income levels, leisure-time physical activity, smoking status, drinking status and hypertension status. The observed prevalences of IFG were plotted and stratified by sleep quality and sleep duration.

Biological interactions should be based on the sum of the scale rather than multiplying the scale. $^{14} 15$ Therefore, we used three measures to estimate biological interactions of poor sleep quality and short sleep duration: relative excess risk owing to interaction (RERI), the attributable proportion (AP) owing to interaction and the synergy index (S). The RERI is the excess risk attributed to interaction relative to the risk without exposure to poor sleep quality and short sleep duration. $\mathrm{AP}$ of disease is caused by interaction in individuals with exposure to both variables. $\mathrm{S}$ is the excess risk from exposure to both variables when there is a biological interaction relative to the risk from exposure to both variables without interaction. In the absence of additive interactions, RERI and $\mathrm{AP}$ are equal to $0 .{ }^{16}$ In the current study, RERI $>0, A P>0$ and $\mathrm{S}>0$ indicate statistical significance. A p value $<0.05$ (two tailed) was considered statistically significant.

\section{RESULTS}

\section{General characteristics of participants}

The response rate was $91.3 \%$. Of the 16584 initial participants, 125 did not respond to the sleep items or blood glucose, 1314 did not meet our study criteria, 15145 adults (7557 men and 7588 women) with complete data were included in our analysis. The average sleep duration per night was $7.16 \pm 1.06 \mathrm{~h}$. The average age was 
Table 2 Comparison of characteristics between individuals with poor sleep quality and sleep duration $<6 \mathrm{~h}$ and controls

\begin{tabular}{|c|c|c|c|c|}
\hline \multirow[b]{2}{*}{ Reported variable } & \multirow[b]{2}{*}{ All } & \multicolumn{3}{|c|}{ Poor sleep quality with short sleep time $(<6 \mathrm{~h})$} \\
\hline & & Yes & No & p Value \\
\hline & 15145 & 602 & 14543 & \\
\hline Age (years) & $47.6 \pm 15.1$ & $47.8 \pm 15.5$ & $47.5 \pm 15.1$ & 0.63 \\
\hline Sex (male) & 7557 & $299(3.96 \%)$ & $7258(96.04 \%)$ & 0.91 \\
\hline Rural & 10965 & $435(3.97 \%)$ & $10530(96.03 \%)$ & 0.94 \\
\hline Manual & 10833 & $421(3.89 \%)$ & $10412(96.11 \%)$ & 0.38 \\
\hline Non-manual & 1045 & $44(4.21 \%)$ & $1001(95.79 \%)$ & 0.69 \\
\hline Unemployed & 677 & $35(5.17 \%)$ & $642(94.83 \%)$ & 0.10 \\
\hline Retired & 2590 & $102(3.94 \%)$ & $2488(96.06 \%)$ & 0.92 \\
\hline Marred (living with partners; \%) & 13403 & $535(3.99 \%)$ & $12868(96.01 \%)$ & 0.77 \\
\hline Below high school & 11899 & $470(3.95 \%)$ & 11429 (96.05\%) & 0.76 \\
\hline High school & 1760 & $70(3.98 \%)$ & $1690(96.02 \%)$ & 1 \\
\hline Above high school & 1486 & $62(4.17 \%)$ & $1424(95.83 \%)$ & 0.68 \\
\hline Smoker & 3541 & $145(4.09 \%)$ & $3396(95.91 \%)$ & 0.68 \\
\hline Alcohol use & 2872 & $121(4.21 \%)$ & $2751(98.79 \%)$ & 0.48 \\
\hline Regular exercise & 2559 & $92(3.60 \%)$ & $2467(96.40 \%)$ & 0.28 \\
\hline Family history of diabetes & 483 & $20(4.14 \%)$ & $463(95.86 \%)$ & 0.85 \\
\hline BMI, mean (SD) & $23.9 \pm 4.7$ & $24.0 \pm 4.9$ & $23.9 \pm 4.6$ & 0.60 \\
\hline Central obesity & 4613 & $186(4.03 \%)$ & $4427(95.97 \%)$ & 0.81 \\
\hline Hypertension & 3060 & $131(4.28 \%)$ & $2929(95.72 \%)$ & 0.33 \\
\hline
\end{tabular}

BMI, Body mass index.

47.6 \pm 15.1 years. Among them, 634 had IFG; the remainder had normal glucose tolerance (NGT). The characteristics of the study population are presented in table 1 . The characteristics between individuals with poor sleep quality and sleep duration $<6 \mathrm{~h}$ and controls are presented in table 2. The proportion of volunteers with poor sleep quality was $26 \%$, the proportion with sleep duration $<6 \mathrm{~h}$ was $12.5 \%$, and the proportion with sleep duration $>8 \mathrm{~h}$ was $12.3 \%$. The $6.7 \%$ prevalence of IFG in volunteers with poor sleep quality was higher than that in volunteers with good sleep quality $\left(\chi^{2}=85.98, p<0.001\right)$. Individuals with sleep duration $<6 \mathrm{~h}$ had a higher IFG prevalence compared with individuals with sleep duration of $6-8 \mathrm{~h} \quad\left(7.3 \%\right.$ vs $\left.3.3 \% ; \chi^{2}=72.20, \mathrm{p}<0.001\right)$. Individuals with sleep duration $>8 \mathrm{~h}$ also had higher prevalence of IFG $\left(6.2 \%\right.$ vs $\left.3.3 \% ; \chi^{2}=37.78, \mathrm{p}<0.001\right)$.

\section{Comparison of PSQI scores between the volunteers} with IFG and NGT

Volunteers with IFG had significantly higher global PSQI scores than those with NGT. For all PSQI items except sleep duration, there were significant differences $(p<0.05)$ in PSQI scores between the two groups (table 3) even after adjusted age.

The association of sleep time and quality with IFG

Individuals with short sleep duration or long sleep duration had significantly increased risk of IFG compared with those with good sleep quality and sleep duration of $6-8 \mathrm{~h}$ (OR $2.16,95 \%$ CI 1.33 to 3.47 ; OR $1.89,95 \%$ CI 1.50 to 2.34 ; respectively; all $\mathrm{p}<0.001$ ), after adjusting for confounders (see table 3). Individuals with poor sleep quality had significantly increased risk of IFG compared with those with good

Table 3 Comparison of Pittsburgh Sleep Quality Index scores between individuals with impaired fasting glucose and controls $(\overline{\mathrm{x}} \pm \mathrm{SD})$

\begin{tabular}{|c|c|c|c|c|}
\hline \multirow[b]{2}{*}{ Items } & \multicolumn{2}{|l|}{ IFG } & \multirow[b]{2}{*}{ F value } & \multirow[b]{2}{*}{ p Value } \\
\hline & No & Yes & & \\
\hline Subjective sleep quality & $0.415 \pm 0.013$ & $0.463 \pm 0.03$ & 31.823 & 0.000 \\
\hline Sleep latency & $0.742 \pm 0.004$ & $0.800 \pm 0.016$ & 27.446 & 0.000 \\
\hline Sleep duration & $0.152 \pm 0.002$ & $0.159 \pm 0.001$ & 0.503 & 0.478 \\
\hline Sleep efficiency & $0.444 \pm 0.003$ & $0.321 \pm 0.001$ & 29.452 & 0.000 \\
\hline Sleep disturbance & $0.591 \pm 0.003$ & $0.658 \pm 0.012$ & 28.259 & 0.000 \\
\hline Use of hypnotic & $0.051 \pm 0.002$ & $0.065 \pm 0.007$ & 16.651 & 0.000 \\
\hline Daytime dysfunction & $0.147 \pm 0.011$ & $0.170 \pm 0.002$ & 9.725 & 0.000 \\
\hline Global PSQI scores & $2.302 \pm 0.009$ & $2.495 \pm 0.041$ & 20.957 & 0.000 \\
\hline
\end{tabular}


Table 4 ORs for the association between sleep quality, sleep duration and impaired fasting glucose among participants

\begin{tabular}{|c|c|c|c|c|}
\hline Variables & IFG & No IFG & OR (95\% Cl) & p Value \\
\hline \multicolumn{5}{|c|}{ Sleep quality } \\
\hline Good & 369 & 10840 & 1 & \\
\hline Poor & 265 & 3671 & 1.21 (1.09 to 1.34$)$ & 0.000 \\
\hline \multicolumn{5}{|c|}{ Sleep duration (h) } \\
\hline $6-8$ & 373 & 10919 & 1 & \\
\hline$<6$ & 145 & 1839 & 2.16 (1.33 to 3.47$)$ & 0.000 \\
\hline$>8$ & 116 & 1753 & 1.89 (1.50 to 2.34$)$ & 0.000 \\
\hline
\end{tabular}

sleep quality (OR 1.98, 95\% CI 1.69 to $2.21 ; \mathrm{p}<0.001$ ), after adjusting for confounders (see table 4 ).

\section{Biological interaction of sleep quality and sleep duration on the prevalence of IFG}

We used a combined effects method to assess interaction, with the $p$ value of the interaction term indicating statistical significance of addictive interactions. Individuals with poor sleep quality or short sleep duration had significantly increased risk of IFG compared with those with good sleep quality and sleep duration of $6-8 \mathrm{~h}$ (OR 2.48, 95\% CI 2.12 to 3.03 ; OR $2.79,95 \%$ CI 2.19 to 3.58 ; respectively; all $p<0.001$ ), after adjusting for confounders. Table 5 shows the results from the multiple logistic regression models. The prevalence of IFG was greatest in those with poor sleep quality and short sleep duration (OR 6.37, 95\% CI 4.66 to 8.67; $\mathrm{p}<0.001$ ), after adjusting for confounders. In addition, individuals with long sleep duration had a significantly increased risk of IFG compared with those who had good sleep quality and sleep duration of $6-8 \mathrm{~h}$ (OR $2.37,95 \%$ CI 1.89 to $2.96 ; \mathrm{p}<0.001$ ), after adjusting for confounders. The prevalence of IFG was also greater in those with good sleep quality with long sleep duration (OR 3.17, 95\% CI 2.29 to $4.41 ; \mathrm{p}<0.001$ ), compared with those with $6-8 \mathrm{~h}$ sleep duration, after adjusting for confounders.

\section{Sensitivity analysis}

There was a strong additive interaction between poor sleep quality and short sleep duration (RERI 1.69, 95\%
CI 0.31 to 3.76.), with $42 \%$ of occurring IFG attributed to the interaction between poor sleep quality and short sleep duration (table 5). There was also interaction between good sleep quality and long sleep duration (RERI $0.78,95 \%$ CI 0.12 to 1.43 ), with $61 \%$ of occurring IFG attributed to the interaction between good sleep quality and long sleep duration (table 6).

\section{DISCUSSION}

The two main findings of this study were, first, that there is combined interaction of poor sleep quality and short sleep duration on the prevalence of IFG. Second, that total PSQI scores of volunteers with IFG were higher than those of volunteers with NGT. We also found that poor sleep quality and short or long sleep duration increased the risk for IFG in a Chinese population, independent of potential confounders such as age, obesity, family history of diabetes, alcohol consumption, smoking, physical activity and other diseases.

Numerous epidemiological studies have demonstrated associations between short or long sleep duration and sleep disturbances and diabetes. ${ }^{17-23}$ However, few articles focused on IFG, and the results of these are inconsistent. ${ }^{524}$ Rafalson et at reported the OR of IFG among short sleepers was 3.0 (95\% CI 1.05 to 8.59) compared with mid-range sleepers with multivariate conditional logistic regression after adjustment for several diabetes risk factors. Hung et $a l^{24}$ reported no association between IFG and poor sleepers. Our findings are consistent with the report by Rafalson $e t$ al.

The global PSQI scores in volunteers with IFG were higher than those in volunteers with NGT, which was in consistent with the Hung $e t a t^{24}$ report. However, there was no difference in sleep duration scores between the volunteers with IFG and the volunteers with NGT. This could be attributed to the fact that $18.3 \%(116 / 634)$ of the volunteers with IFG were long sleepers, while short sleepers with IFG only accounted for $22.9 \%$ (145/634).

Short sleep duration increases appetite. After four nights of $4.5 \mathrm{~h}$ in bed, compared with after four nights of $8.5 \mathrm{~h}$ in bed, caloric intake, especially of carbohydrate-rich nutrients, increased by $14 \%$ in young normal-weight men and women. ${ }^{25}{ }^{26}$ Middle-aged obese volunteers submitted to four to five nights of restriction of their habitual sleep schedule by $2-3 \mathrm{~h} /$ night ate $15 \%$

Table 5 ORs for the association between sleep quality and impaired fasting glucose by sleep duration among participants

\begin{tabular}{llrrrr}
\hline Sleep duration & Sleep quality & IFG & No IFG & OR (95\% Cl) & p Value \\
\hline $6-8 \mathrm{~h}$ & Good & 246 & 8743 & 1 & \\
& Poor & 127 & 2176 & $1.98(1.76$ to 2.52) & $<0.001$ \\
$<6 \mathrm{~h}$ & Good & 53 & 1329 & $1.38(1.12$ to 1.61$)$ & $<0.001$ \\
& Poor & 92 & 510 & $6.37(4.66$ to 8.67$)$ & $<0.001$ \\
& Good & 70 & 768 & $3.17(2.29$ to 4.41$)$ & $<0.001$ \\
& Poor & 46 & 985 & $1.59(1.08$ to 2.29) & $<0.001$ \\
\hline
\end{tabular}

Adjusted for age, sex, education, employment, BMI, family history of diabetes, smoking status, alcohol consumption and physical activity. BMI, body mass index; IFG, impaired fasting glucose. 
Table 6 Measures for estimation of the biological interaction between sleep quality and sleep duration for the prevalence of impaired fasting glucose in participants

\section{Measures of biological} interaction

Estimate (95\% Cl)

\begin{tabular}{lc} 
Poor sleep quality versus sleep duration $<6 \mathrm{~h}$ \\
RERI & $1.69(0.31$ to 3.76$)$ \\
AP & $0.42(0.15$ to 0.61$)$ \\
S & $2.85(2.14$ to 3.92$)$ \\
Good sleep quality versus sleep duration $>8 \mathrm{~h}$ \\
RERI & $0.78(0.12$ to 1.43$)$ \\
AP & $0.61(0.26$ to 0.87$)$ \\
S & $-0.65(-0.94$ to -0.27$)$ \\
\hline
\end{tabular}

Reference group is good sleep quality with $6-8 \mathrm{~h}$ sleep duration. Adjusted for age, sex, education, occupation, BMI, family history of diabetes, smoking status, alcohol consumption and hypertension.

AP, attributable proportion; BMI, body mass index; RERI, relative excess risk of interaction; S, synergy index.

more calories than those whose habitual sleep schedule was extended by the same amount. ${ }^{27}$ In volunteers with sleep debt, glucose tolerance, glucose effectiveness and insulin sensitivity are decreased, and $\beta$-cell function is reduced. ${ }^{6} 2628{ }^{29}$ Sleep loss also results in decreased anorexigenic leptin levels, ${ }^{30-32}$ especially in volunteers with chronic sleep restriction. ${ }^{33}$ Observational epidemiological studies have also shown reduced leptin levels, after controlling for BMI or adiposity, in habitual short sleepers. ${ }^{33}$ Conversely, leptin deficiency disrupts sleep architecture and impairs sleep consolidation. ${ }^{35}$ Taken together, these data suggest that sleep loss is likely to have a profound impact on IFG.

Poor sleep quality, independent of sleep duration, impairs glucose regulation in healthy young adults. ${ }^{36} 37$ After three nights of slow-wave sleep suppression, insulin sensitivity is decreased by $\sim 25 \%$, reaching the level reported in older adults and in populations at high risk of diabetes. ${ }^{38}$ The decrease in insulin sensitivity is not compensated for by an increase in insulin release. Consequently, the disposition index is $\sim 20 \%$ lower. Consistent with an increased diabetes risk, glucose tolerance is reduced by $\sim 23 \%$ reaching the range typical of older adults with IGT. $^{37}$ Sleep fragmentation also increases sympathetic activity, which in turn leads to disorders of glucose metabolism. ${ }^{39}$

Taken together, short sleep duration, increased caloric intake, poor sleep quality, decreased leptin levels, decreased insulin sensitivity and increased sympathetic activity affect each other, creating a vicious circle, which elevates the risk of IFG.

Despite the absence of the synergistic effect of goodquality sleep with long sleep duration, a strong association of IFG with long sleep duration in individuals was observed, suggesting an independent effect of long sleep duration.

The strengths of this study were that participants of the large sample were randomly selected from the general population of Xuzhou, and many confounding risk factors were adjusted for. There were three notable limitations. Firstly, because of the cross-sectional design, we could not determine a causal relationship between sleep quality, sleep duration and IFG. Secondly, we were not able to control for some important and well-known risk factors of diabetes, for example, snoring. ${ }^{40}$ Thirdly, we did not measure poor diet, which is causally related to type 2 diabetes and may also influence sleep patterns. ${ }^{41} 42$

In summary, volunteers who experience short sleep durations with poor sleep quality are six times more likely to develop IFG than those whose average sleep was $6-8 \mathrm{~h}$ a night with good sleep quality. The joint effect of short sleep duration and poor sleep quality was positive.

Acknowledgements This research was funded by the Preventive Medicine research projects of Jiangsu Province Health Department in 2012 (Y2012025) and the Science and Technology projects of Xuzhou City in 2011 (XF11C090). Double-Hundred Talents Project of Xuzhou city in 2012 (BRA201224).

Funding This research received no specific grant from any funding agency in the public, commercial or not-for-profit sectors.

Competing interests None.

Patient consent Obtained.

Ethics approval The study protocol was approved by the Xuzhou Center for Disease Control and Prevention.

Provenance and peer review Not commissioned; externally peer reviewed.

Data sharing statement No additional data are available.

Open Access This is an Open Access article distributed in accordance with the Creative Commons Attribution Non Commercial (CC BY-NC 3.0) license, which permits others to distribute, remix, adapt, build upon this work noncommercially, and license their derivative works on different terms, provided the original work is properly cited and the use is non-commercial. See: http:// creativecommons.org/licenses/by-nc/3.0/

\section{REFERENCES}

1. Yang W, Lu J, Weng J, et al. China National Diabetes and Metabolic Disorders Study Group. Prevalence of diabetes among men and women in China. N Engl J Med 2010;362:1090-101.

2. Li G, Zhang P, Wang J, et al. The long-term effect of lifestyle interventions to prevent diabetes in the China Da Qing Diabetes Prevention Study: a 20-year follow-up study. Lancet 2008;371:1783-9.

3. Lou P, Chen P, Yu J, et al. Analysis on the risk factors of the residents with impaired fasting glucose in Xuzhou. Chin J Dis Control Prev 2011;15:192-5. (In Chinese)

4. Najafian J, Mohamadifard N, Siadat ZD, et al. Association between sleep duration and diabetes mellitus: Isfahan Healthy Heart Program. Niger J Clin Pract 2013;16:59-62.

5. Rafalson L, Donahue RP, Stranges S, et al. Short sleep duration is associated with the development of impaired fasting glucose: the Western New York Health Study. Ann Epidemiol 2010;20:883-9.

6. Spiegel K, Leproult R, Van Cauter E. Impact of sleep debt on metabolic and endocrine function. Lancet 1999;354:1435-9.

7. Lou $\mathrm{P}$, Chen $\mathrm{P}$, Zhang L, et al. Relation study of sleep quality and sleep duration to type 2 diabetes: a population-based cross-sectional survey. BMJ Open 2012;2:e000956.

8. Wu $\mathrm{H}$, Lou $\mathrm{P}$, Chen $\mathrm{P}$, et al. Survey the relationship between sleep quality and T2DM. Chin J Diabetes 2013;21:330-3. (In Chinese)

9. Chen $\mathrm{P}$, Lou $\mathrm{P}, \mathrm{Yu}$ J, et al. Risk factors of diabetes mellitus of residents living in Xuzhou city. Chin J Health Manage 2010;4:78-80. (In Chinese)

10. Buysse DJ, Reynolds CF III, Monk TH, et al. The Pittsburgh Sleep Quality Index: a new instrument for psychiatric practice and research. Psychiatry Res 1989;28:193-213.

11. American Diabetes Association. Diagnosis and classification of diabetes mellitus. Diabetes Care 2010;33(Suppl 1):S62-9. 
12. Tsai PS, Wang SY, Wang MY, et al. Psychometric evaluation of the Chinese version of the Pittsburgh Sleep Quality Index (CPSQI) in primary insomnia and control subjects. Qual Life Res 2005; 14:1943-52.

13. Cooperative Meta-analysis Group of China Obesity Task Force. Predictive values of body mass index and waist circumference to risk factors of related disease in Chinese adult population. Chin $J$ Epidemiol 2002;23:5-10.

14. Rothman KJ, Greenland S, Lash TL. Modern epidemiology. 3rd edn. Philadelphia: Lippincott Williams \& Wilkins, 2008.

15. Hosmer DW, Lemeshow S. Confidence interval estimation of interaction. Epidemiology 1992;3:452-6.

16. Knol MJ, VanderWeele TJ, Groenwold RHH, et al. Estimating measures of interaction on an additive scale for preventive exposures. Eur J Epidemiol 2011;26:433-8.

17. Gottlieb DJ, Punjabi NM, Newman AB, et al. Association of sleep time with diabetes mellitus and impaired glucose tolerance. Arch Intern Med 2005;165:863-8.

18. Yaggi HK, Araujo AB, McKinlay JB. Sleep duration as a risk factor for the development of type 2 diabetes. Diabetes Care 2006;29: 657-61.

19. Hayashino Y, Fukuhara S, Suzukamo Y, et al. Relation between sleep quality and quantity, quality of life, and risk of developing diabetes in healthy workers in Japan: the High-risk and Population Strategy for Occupational Health Promotion (HIPOP-OHP)Study. BMC Public Health 2007;7:129.

20. Shankar A, Syamala S, Kalidindi S. Insufficient rest or sleep and its relation to cardiovascular disease, diabetes and obesity in a national, multiethnic sample. PLoS One 2010;5:e14189.

21. Mallon L, Broman JE, Hetta J. High incidence of diabetes in men with sleep complaints or short sleep duration-a 12-year follow-up study of a middle-aged population. Diabetes Care 2005;28: 2762-7.

22. Nilsson PM, RÖÖst M, EngstrÖm M, et al. Incidence of diabetes in middle-aged men is related to sleep disturbances. Diabetes Care 2004:27:2464-9.

23. Vgontzas AN, Liao D, Pejovic S, et al. Insomnia with objective short sleep duration is associated with type 2 diabetes: a population-based study. Diabetes Care 2009;32:1980-5

24. Hung HC, Yang YC, Ou HY, et al. The relationship between impaired fasting glucose and self-reported sleep quality in a Chinese population. Clin Endocrinol (Oxf) 2013;78:518-24.

25. Tasali E, Leproult R, Spiegel K. Reduced sleep duration or quality: relationships with insulin resistance and type 2 diabetes. Prog Cardiovasc Dis 2009;51:381-91.

26. Nedeltcheva AV, Kessler L, Imperial J, et al. Exposure to recurrent sleep restriction in the setting of high caloric intake and physical inactivity results in increased insulin resistance and reduced glucose tolerance. J Clin Endocrinol Metabol 2009:94:3242-50.
27. Morselli $\mathrm{L}$, Balbo $\mathrm{M}$, Van Cauter $\mathrm{E}$, et al. Impact of sleep restriction on the regulation of appetite in middle-aged obese subjects. 4th International World Sleep Congress; Quebec City, Québec, 2011.

28. Buxton OM, Pavlova M, Reid EW, et al. Sleep restriction for 1 week reduces insulin sensitivity in healthy men. Diabetes 2010;59:2126-33.

29. Simpson NS, Banks S, Dinges DF. Sleep restriction is associated with increased morning plasma leptin concentrations, especially in women. Biol Res Nurs 2010;12:47-53.

30. Guilleminault C, Powell NB, Martinez S, et al. Preliminary observations on the effects of sleep time in a sleep restriction paradigm. Sleep Med 2003;4:177-84.

31. Spiegel K, Leproult R, L'hermite-Balériaux M, et al. Leptin levels are dependent on sleep duration: relationships with sympathovagal balance, carbohydrate regulation, cortisol, and thyrotropin. J Clin Endocrinol Metab 2004;89:5762-71.

32. Spiegel K, Tasali E, Penev $\mathrm{P}$, et al. Sleep curtailment in healthy young men is associated with decreased leptin levels, elevated ghrelin levels, and increased hunger and appetite. Ann Intern Med2004;141: 846-50.

33. Taheri S, Lin L, Austin D, et al. Short sleep duration is associated with reduced leptin, elevated ghrelin, and increased body mass index. PLoS Med 2004;1:e62.

34. Chaput JP, Despres JP, Bouchard C, et al. Short sleep duration is associated with reduced leptin levels and increased adiposity: results from the Quebec family study. Obesity (Silver Spring) 2007;15:253-61.

35. Laposky AD, Shelton J, Bass J, et al. Altered sleep regulation in leptin-deficient mice. Am J Physiol Regul Integr Comp Physiol 2006;290:R894-903.

36. Stamatakis KA, Punjabi NM. Effects of sleep fragmentation on glucose metabolism in normal subjects. Chest 2010;137:95-101.

37. Tasali E, Leproult R, Ehrmann DA, et al. Slow-wave sleep and the risk of type 2 diabetes in humans. Proc Natl Acad Sci USA 2008;105:1044-9.

38. Bergman RN. Lilly lecture 1989. Toward physiological understanding of glucose tolerance. Minimal-model approach. Diabetes 1989;38:1512-27.

39. Peltier AC, Consens FB, Sheikh K, et al. Autonomic dysfunction in obstructive sleep apnea is associated with impaired glucose regulation. Sleep Med 2007;8:149-55.

40. Al-Delaimy WK, Manson JE, Willett WC, et al. Snoring as a risk factor for type II diabetes mellitus: a prospective study. $\mathrm{Am} \mathrm{J}$ Epidemiol 2002;155:387-93.

41. Tonstad SMD, Butler T, Yan R, et al. Type of vegetarian diet, body weight, and prevalence of type 2 diabetes. Diabetes Care 2009;32:791-6.

42. Grandner MA, Kripke DF, Naidoo N, et al. Langer relationships among dietary nutrients and subjective sleep, objective sleep, and napping in women. Sleep Med 2010;11:180. 